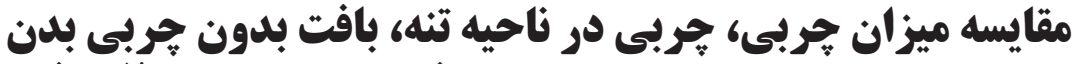

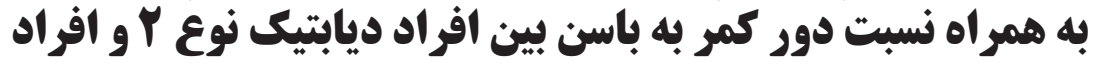

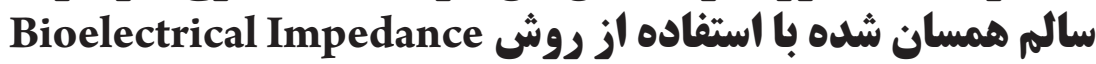

\section{Analysis}

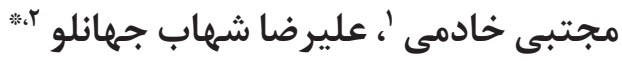

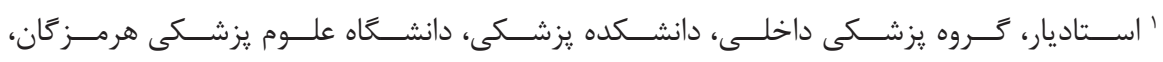

$$
\text { بندرعبــاس، ايــــان }
$$

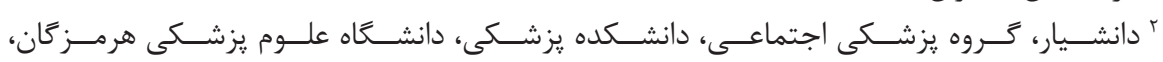

$$
\text { بندرعبـاس، ايــران }
$$

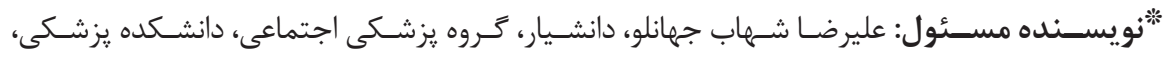

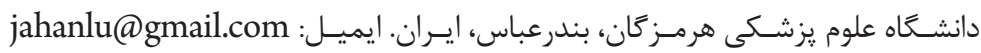

DOI: $10.21859 /$ hums-24017

קقكيده

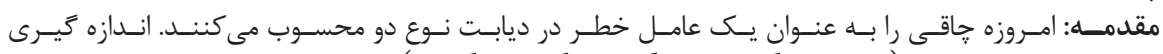

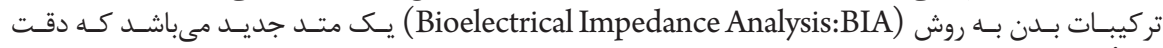

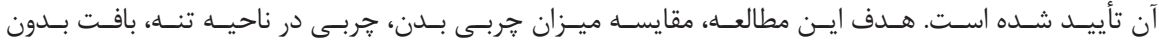

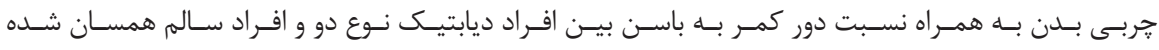
اسـت

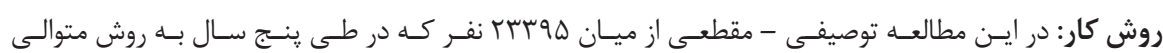

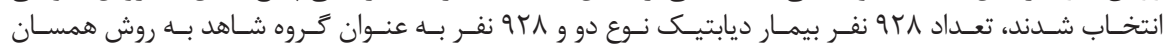

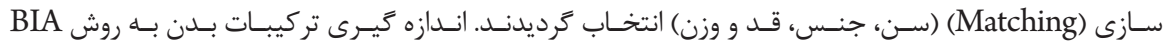

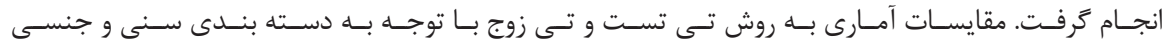

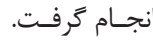

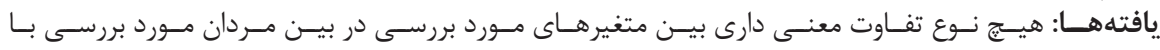

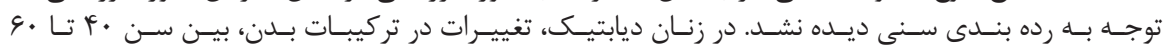

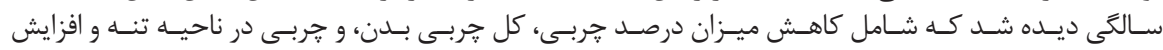

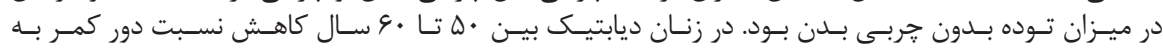

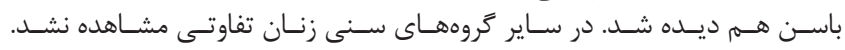

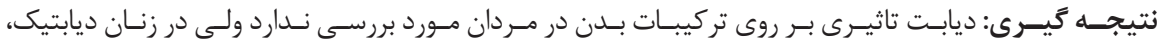

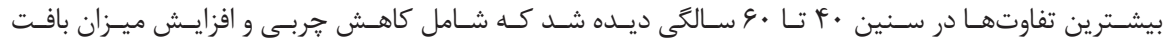

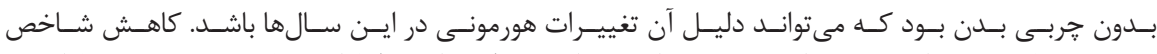

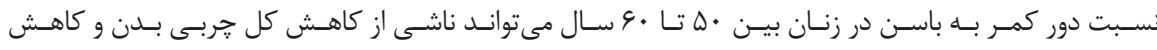

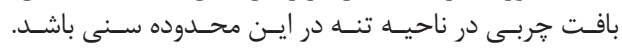

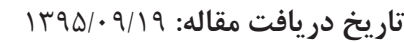

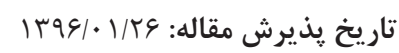

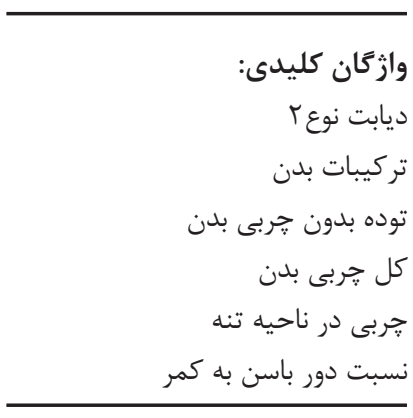

تمامى حقوق نشر براى دانشكاه علوم يزشكى همدان محفوظ است.

مقدمه

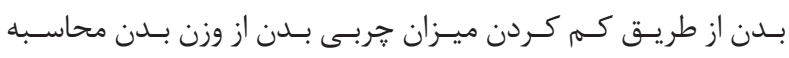

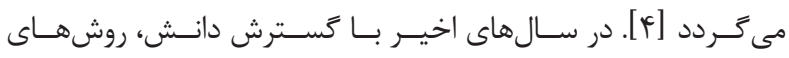

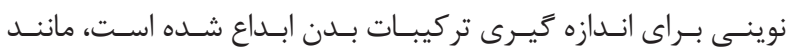
Magnetic Resonance Imaging (MRI)

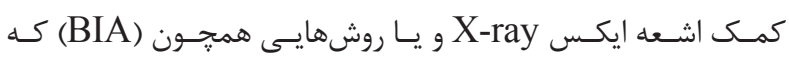

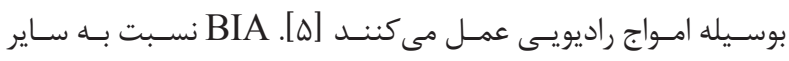

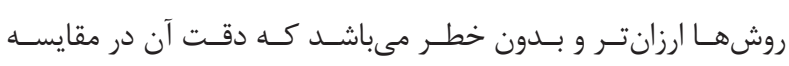

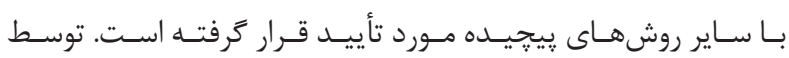

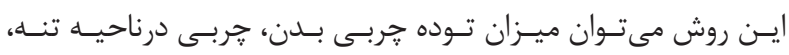

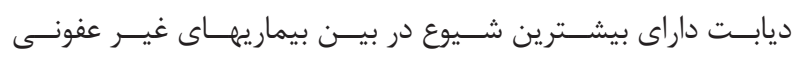

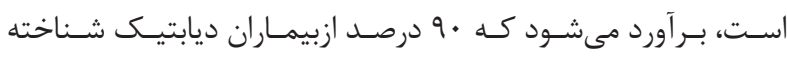

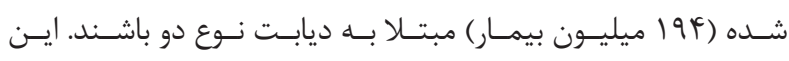

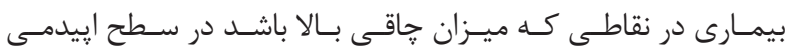

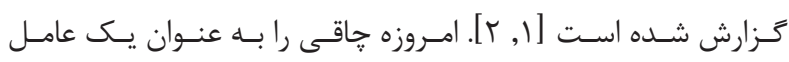

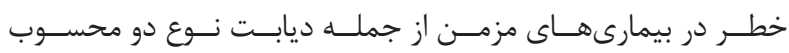

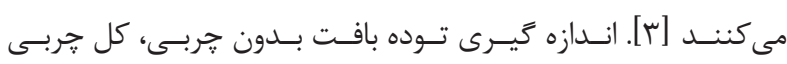

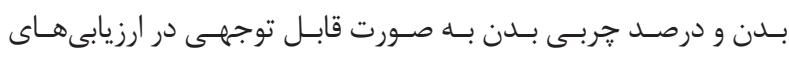

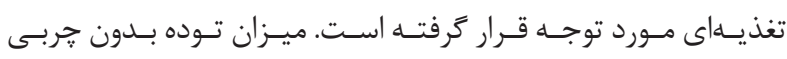




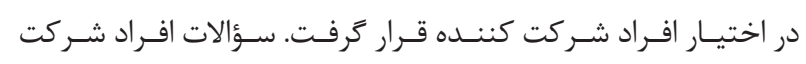

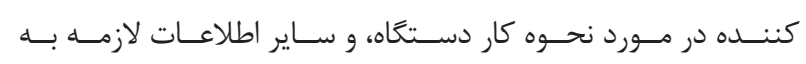

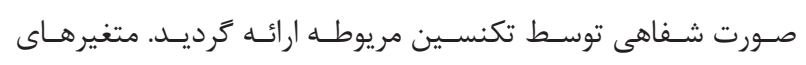

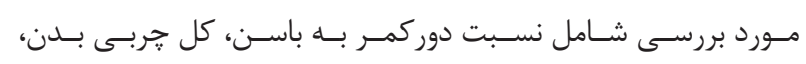

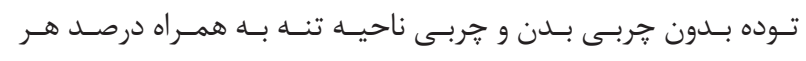

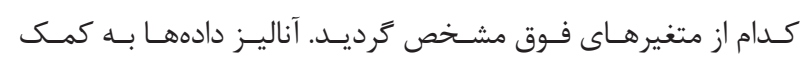

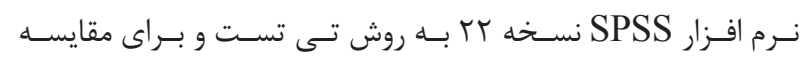

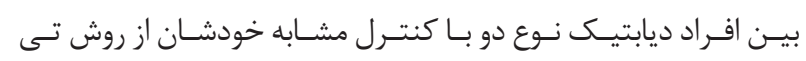

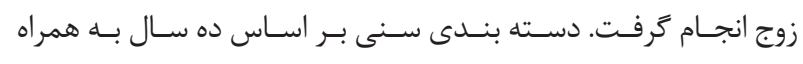

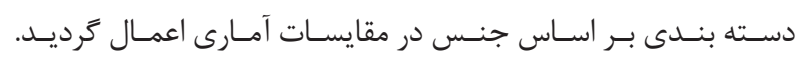

\section{بافته ها}

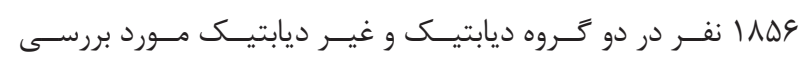

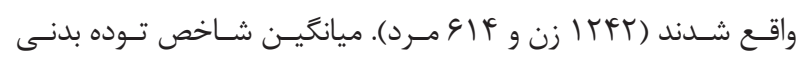

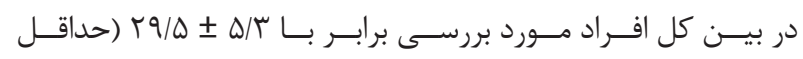

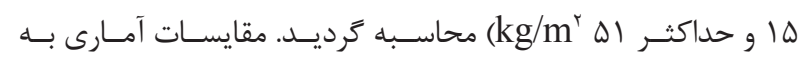

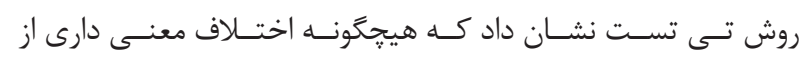

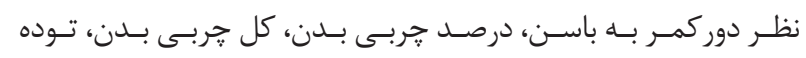

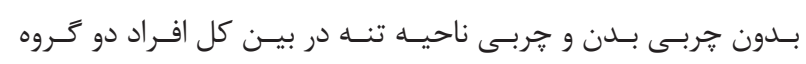

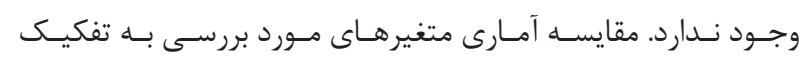

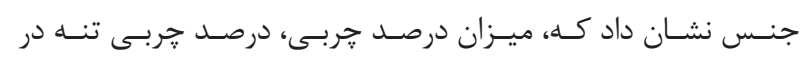

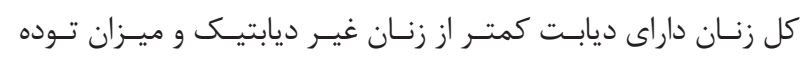

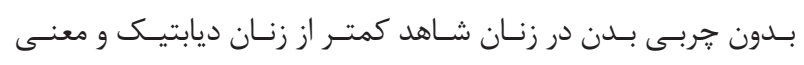

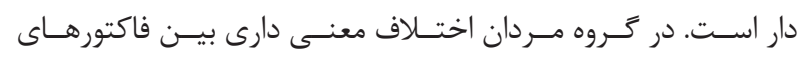

مــورد بررسـى ديسده نشـد (جـدول () ).

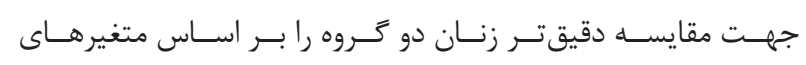

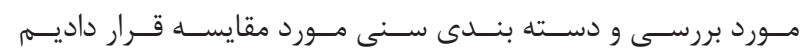

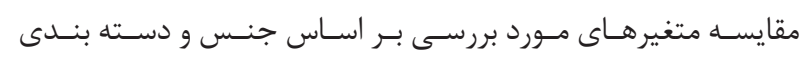

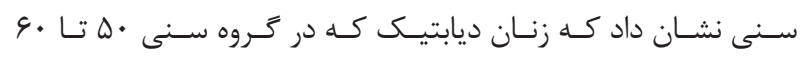

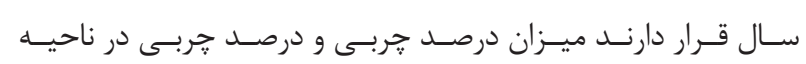

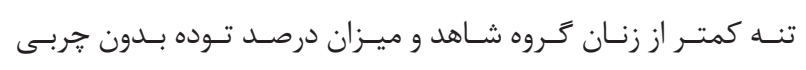

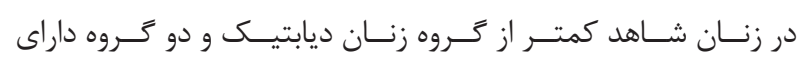

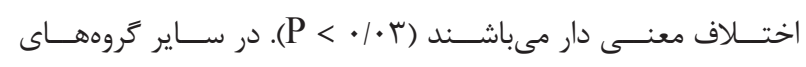

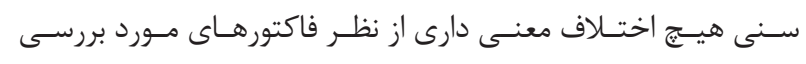

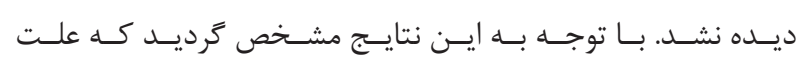

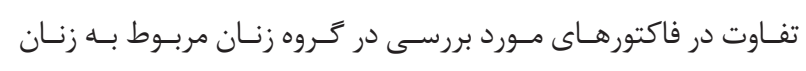

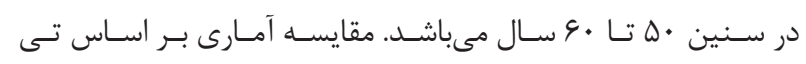

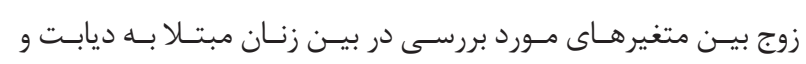

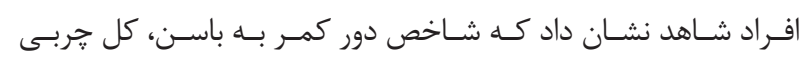

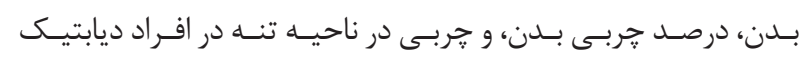

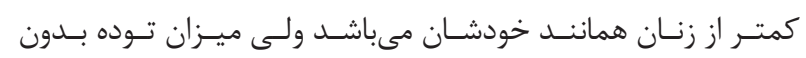

درصـد جربسى بــن و ميـزان بافـت بـدون جربسى بــن ران را محاسـبه

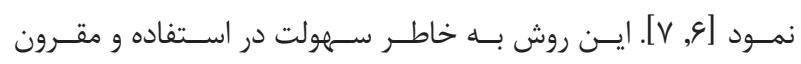

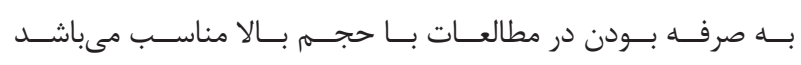

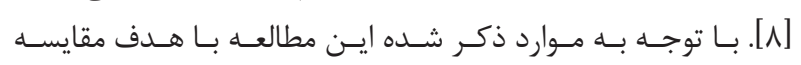

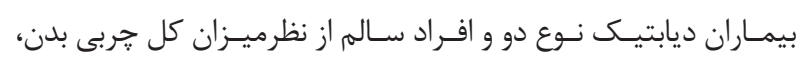

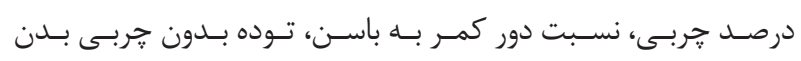

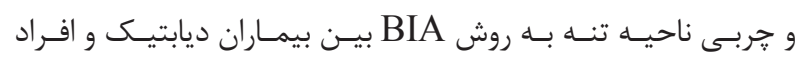

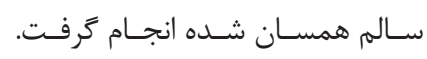

\section{روش كار}

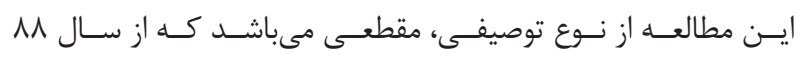

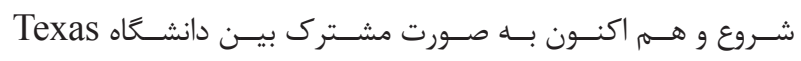
A\&M University

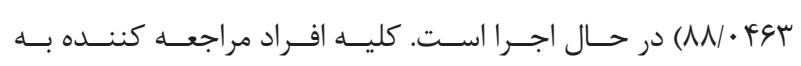

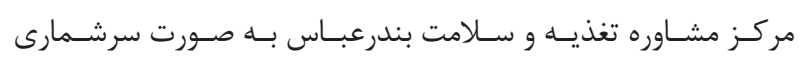

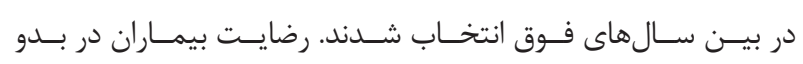

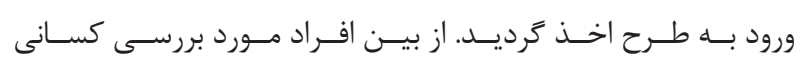

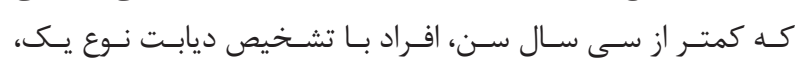

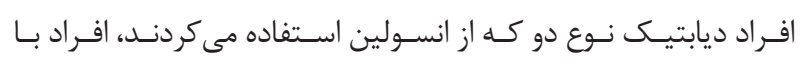

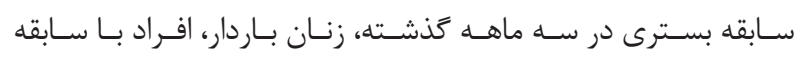

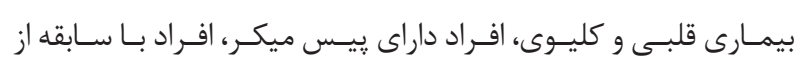

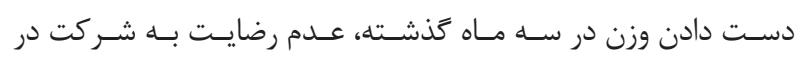

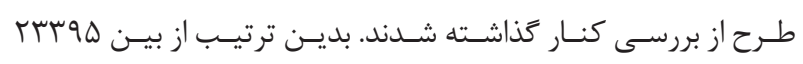

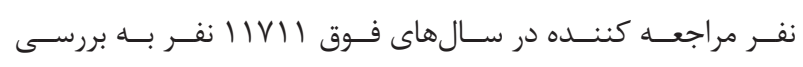

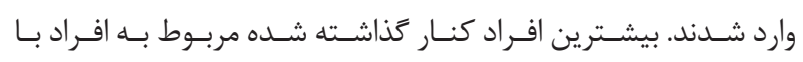

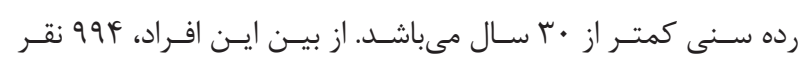

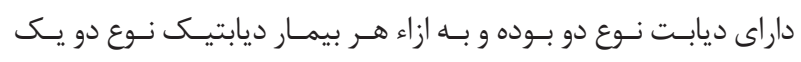

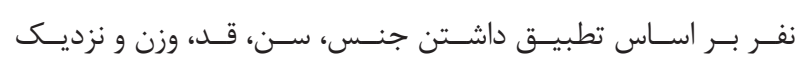

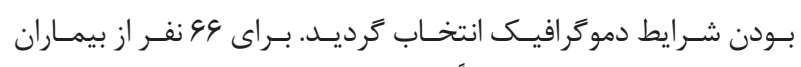

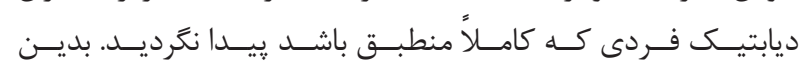

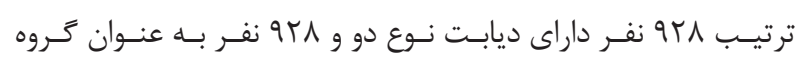

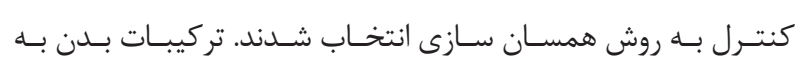

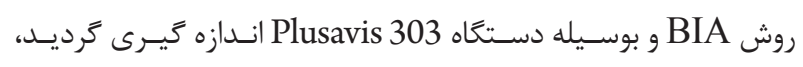

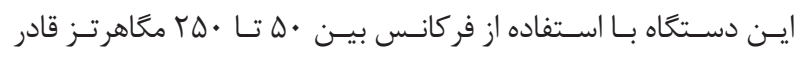

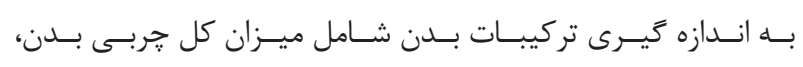

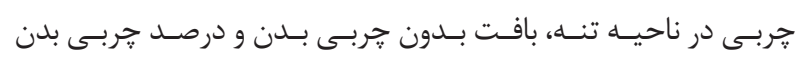

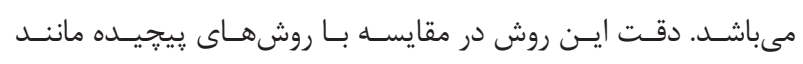

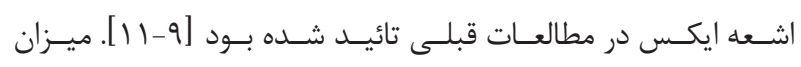

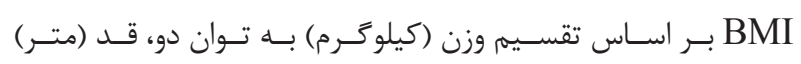

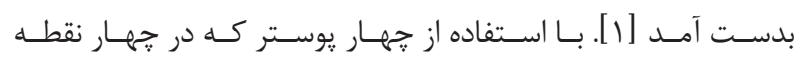
مركـز نصـب شـده بود بـه همـراه بروشـورهاى رايـعان، اطلاعـات لازم 


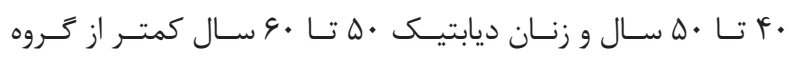

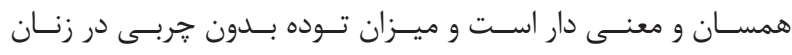

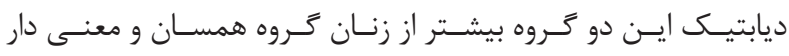

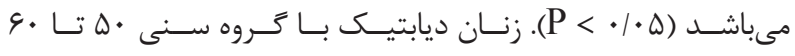

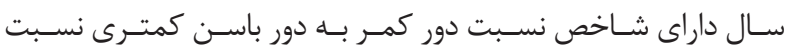

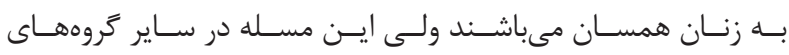

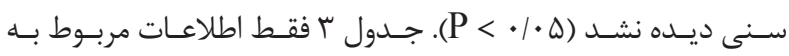

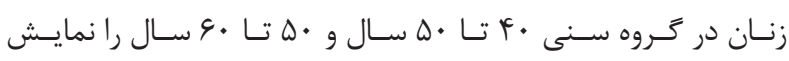

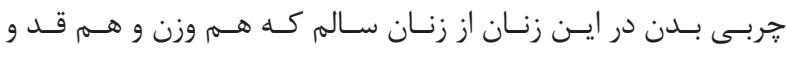

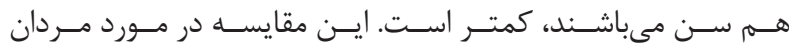

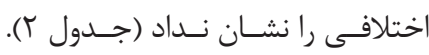

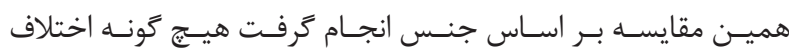

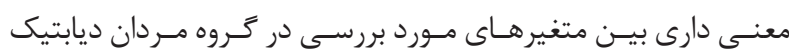

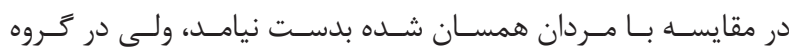

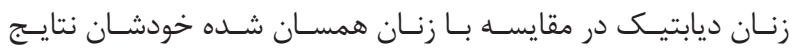

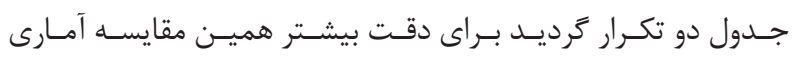

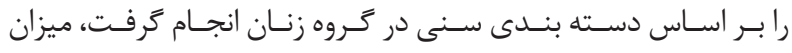

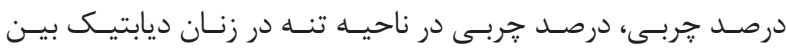

\begin{tabular}{|c|c|c|c|c|c|}
\hline \multirow[b]{2}{*}{$\begin{array}{l}\text { درصد جربى ناحيه تنه } \\
\text { Mean } \pm \text { SD }\end{array}$} & \multirow[b]{2}{*}{$\begin{array}{c}\text { درصد توده بدون جربى بدن } \\
\text { Mean I SD }\end{array}$} & \multicolumn{4}{|c|}{ جدول ا: مقايسه بر اساس فاكتورهاى مورد بررسى در كل افراد و به تفكيك جنس } \\
\hline & & درصد جربى بدن & نسبت دور كمر به باسن & تعداد & متغير \\
\hline $19 / 9 \pm r / \Lambda$ & $\varepsilon V / 1 \pm V / \Delta$ & $r t / \Lambda \pm \cdot / V$ & $\cdot / 9 \cdot r \pm \cdot / V$ & $9 r \wedge$ & كل افراد ديابتيك \\
\hline$I V / r \pm r / V$ & $99 / 0 \pm V / r$ & $r r / \mathcal{A} \pm \cdot / V$ & $\cdot / 9 \cdot 1 \pm \cdot / V$ & $94 \wedge$ & كل افراد شاهد \\
\hline$\cdot / \cdot V_{*}$ & $\cdot / \cdot V_{*}$ & $\cdot / \cdot \Lambda *$ & $\cdot / \cdot \wedge *$ & & P ارزش \\
\hline$|r /| \pm r / \mid$ & $V F / 4 \pm 9$, & $r \Delta / \Delta \pm \cdot / \varphi$ & $\cdot / 941 \pm \cdot / V$ & $r \cdot v$ & مردان ديابتيك \\
\hline $\mid r / \varphi \pm r / \cdot$ & $V \Psi / 9 \pm \Delta / 9$ & $\lceil 9 / \cdot \pm \cdot \mid \Delta$ & $\cdot / 949 \pm \cdot / V$ & $r \cdot v$ & مردان غير ديابتيك \\
\hline$\cdot / r 9 *$ & $\cdot / 49 *$ & $\cdot / T V *$ & $\cdot / \Gamma V *$ & & ارزش P \\
\hline $1 N / \Lambda \pm r / 9$ & 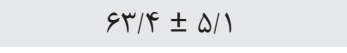 & $r \varepsilon / \Delta \pm \Delta / \cdot * * *$ & $\cdot \mid \Lambda \Lambda \mu \pm \cdot / 9$ & STI & زنان ديابتيك \\
\hline $19 / 1 \pm r / 4$ & $G r / \Lambda \pm F / V$ & $r V / l \pm f / V_{*} * *$ & $\cdot / 1 \wedge 9 \pm \cdot / \Delta$ & GTI & زنان غير ديابتيك \\
\hline$\cdot / \cdot r * * *$ & $\cdot / \cdot r * * *$ & . $/ . r_{*} * *$ & $.19 * *$ & & P ارزش \\
\hline
\end{tabular}

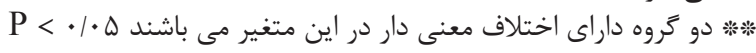

\begin{tabular}{|c|c|c|c|c|}
\hline \multirow[b]{2}{*}{ P ارزش P } & \multirow[b]{2}{*}{ زنان غير ديابتيك } & \multicolumn{3}{|c|}{ جدول r: مقايسه شاخصهاى مورد بررسى در زنان شاهد و ديابتيك به روش تى زوجى } \\
\hline & & زنان ديابتيك & تعداد & وضعيت \\
\hline$<\cdot 1 \cdot 1$ & $\cdot / 1 \wedge 9 \pm \cdot / \cdot \Delta$ & $\cdot \mid \Lambda \Lambda \mu \pm \cdot / \cdot \varphi$ & GTI & 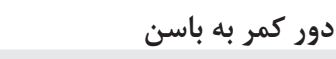 \\
\hline$<\cdot / \cdot 1$ & $r \Lambda / V \pm \Lambda / \mathcal{F}$ & $r \Lambda / r \pm \Lambda / V$ & GTI & كل جربى بدن (kg) (kg) \\
\hline$<\cdot / \cdot 1$ & $r V / I \pm F / V$ & $r q / \Delta \pm \Delta / \cdot$ & arl & درصد جربى بدن (\%) \\
\hline$<\cdot 1 \cdot 1$ & $\| F / \Lambda \pm f / r$ & $\mid f / \Delta \pm F / \Delta$ & GTI & جربى در ناحيه تنه (kg) \\
\hline$<\cdot / \cdot 1$ & $F V / T \pm g / V$ & $F V / 9 \pm 9 / 1$ & Gri & توده بدون جربى بدن (kg) \\
\hline
\end{tabular}

\begin{tabular}{|c|c|c|c|c|c|}
\hline \multirow[b]{2}{*}{ درصد جربى ناحيه تنه ** } & \multirow{2}{*}{ در شده بر اساس دسته بندى سنى } & \multirow{2}{*}{ دايسه با زنان سالم همس جرب بدن } & \multirow{2}{*}{ نسبت دور كمر به باسن * ث } & \multirow{2}{*}{ 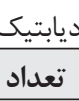 } & \multirow{2}{*}{ متغول } \\
\hline & & & & & \\
\hline & & & & & 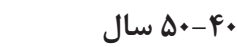 \\
\hline $1 N / 9 \pm r / 4$ & $\Delta V / \varphi \pm F / \varphi$ & $r \varepsilon / 9 \pm r / \varphi$ & $\cdot \mid \Lambda \Lambda T \pm \cdot / \cdot \Delta$ & $r \cdot \Lambda$ & زنان غير ديابتيك \\
\hline $1 N / 9 \pm T / \Delta$ & $\Delta \Lambda / r \pm r / q$ & $\lceil 4 / \mid \pm \Delta / \cdot$ & $\cdot \mid \wedge \vee \wedge \pm \cdot / \cdot \Delta$ & $r \cdot \Lambda$ & زنان ديابتيك \\
\hline \multirow[t]{2}{*}{$<\cdot 1 \cdot 1$} & $<\cdot / \cdot 1$ & $<\cdot / \cdot 1$ & - & & P ارزش P \\
\hline & & & & & 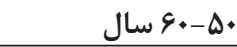 \\
\hline $19 / 4 \pm r / f$ & $\Delta \varphi / \varphi \pm F / \varphi$ & $r V / V \pm F / \varphi$ & $\cdot / 9 \cdot 1 \pm \cdot \cdot \cdot \Delta$ & rm & زنان غير ديابتيك \\
\hline $11 / 9 \pm r / 9$ & $\Delta V / V \pm \Delta / T$ & $r \varepsilon / V \pm \Delta / r$ & $.1194 \pm .1 .9$ & ו ו & زنان ديابتيك \\
\hline$<\cdot 1 \cdot \cdot 1$ & $<\cdot 1 \cdot \cdot 1$ & $<\cdot 1 \cdot \cdot 1$ & $<\cdot 1 \cdot \cdot 1$ & & P P P P P \\
\hline
\end{tabular}

* مقادير جدول بصورت ميانخين \pm انحراف معيار بيان شده اند. 


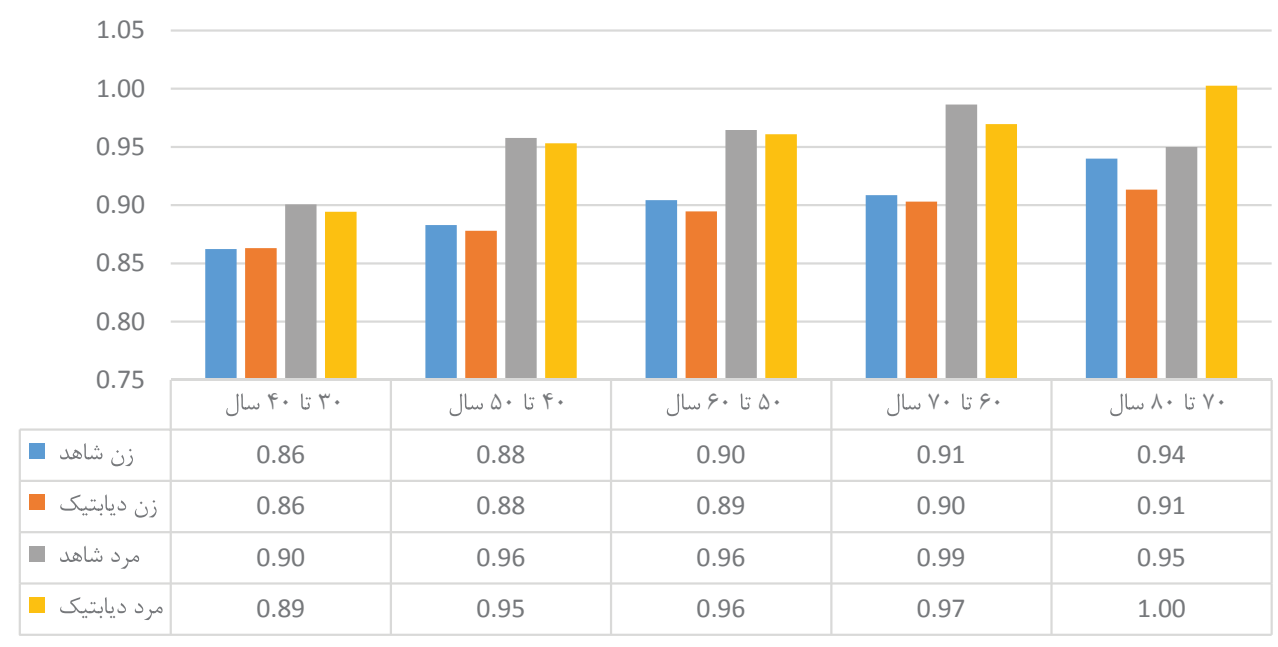

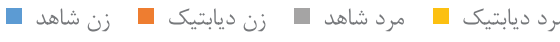

تصوير ا: مقايسه نسبت دور كمر به دور باسن بر اساس دسته بندى سن وجنس در دو كروه شاهد و ديابتيك

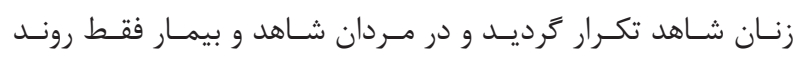

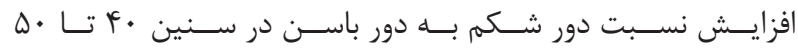

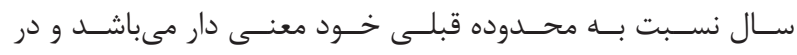

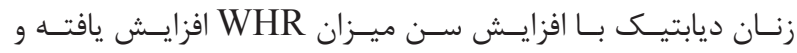

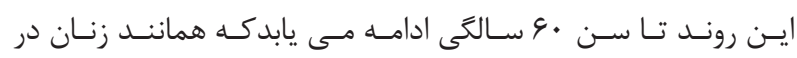

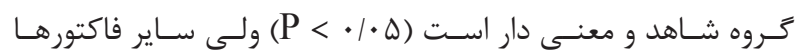

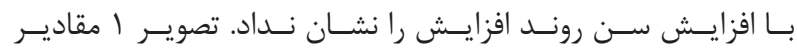

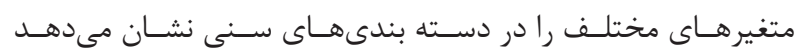

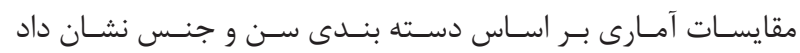

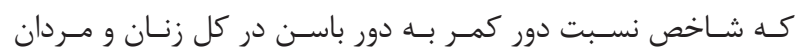

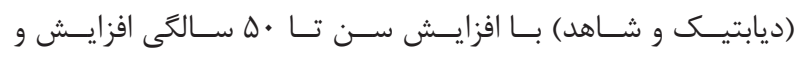

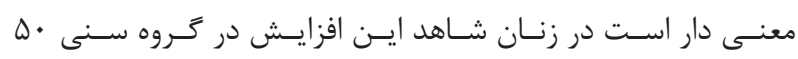

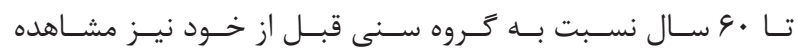

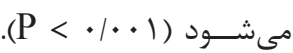

بيمـار ان مـرد ديابتيـك مشـاركت كنـــــه در ايـن طـرح در مقايسـهـ

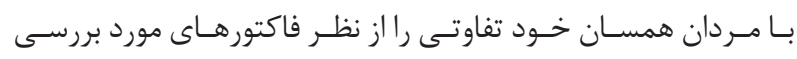

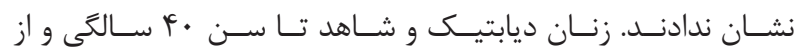

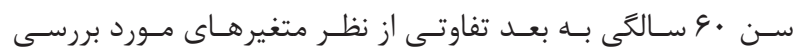

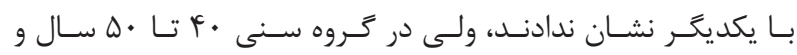

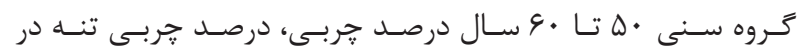

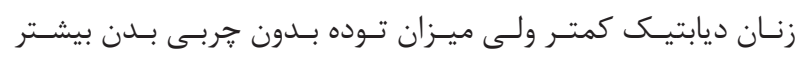

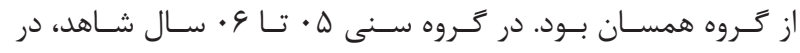

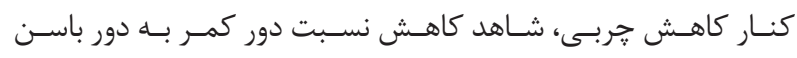

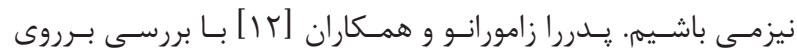

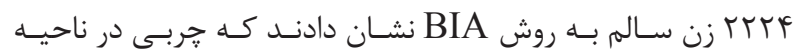

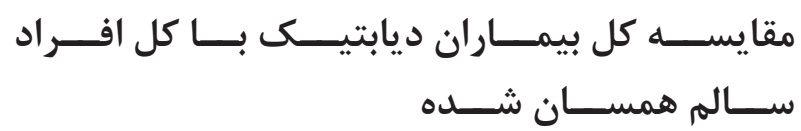

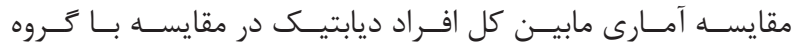

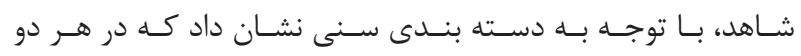

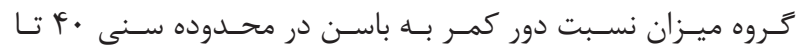

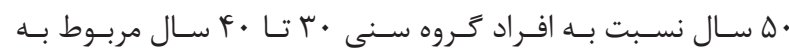

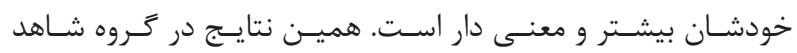

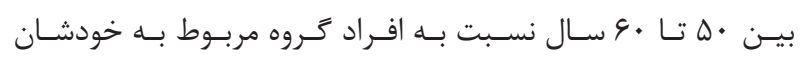

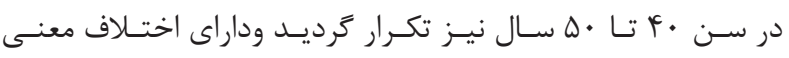

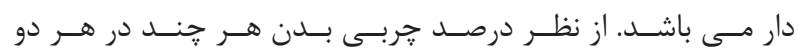

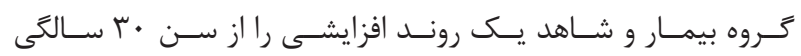

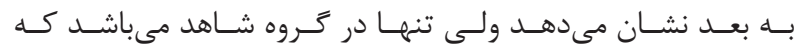

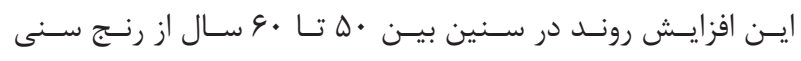

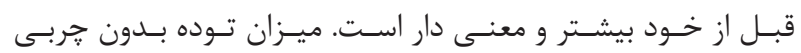

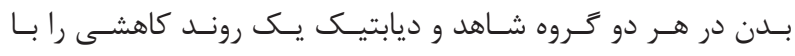

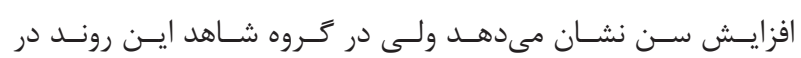

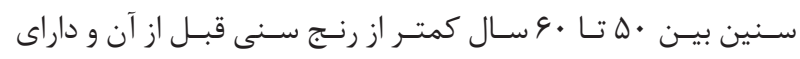

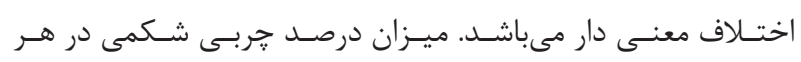

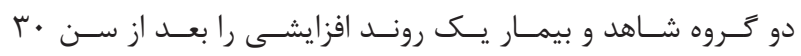

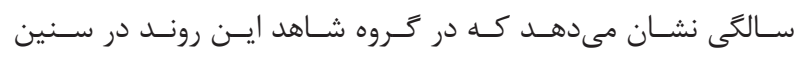

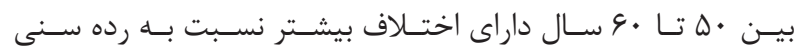

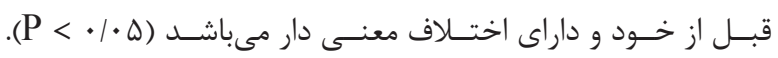

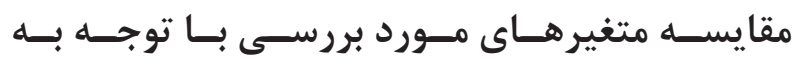

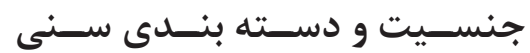
هميـن مقايسـات بـر اسـاس جنـس انجـام كرفــت كليسه نتايـج در 


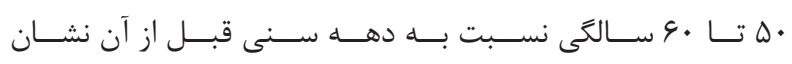

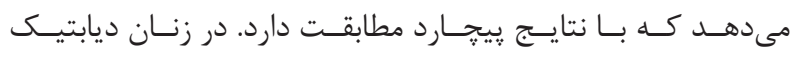

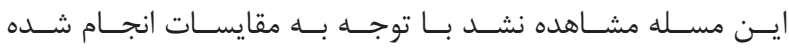

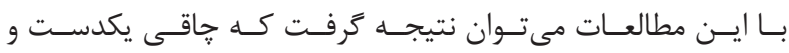

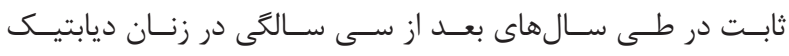

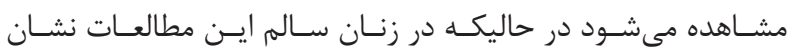

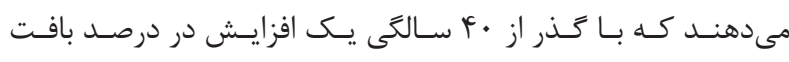

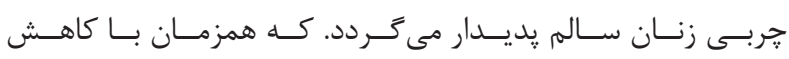

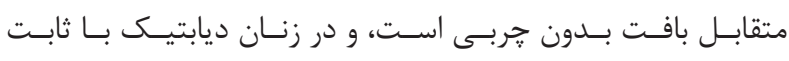

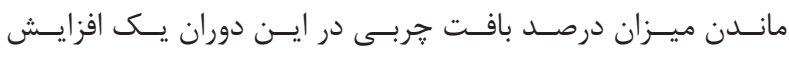

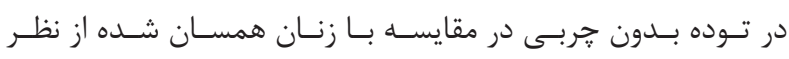

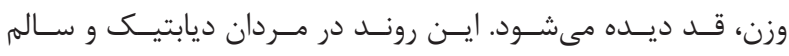

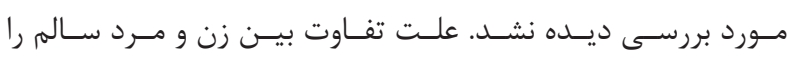

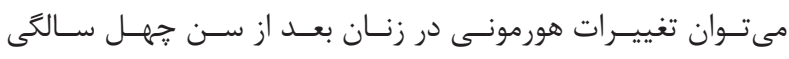

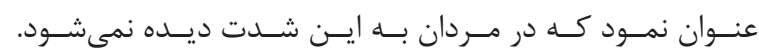

نتيجه تيرى

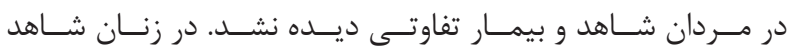

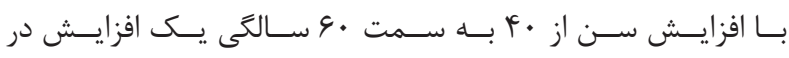

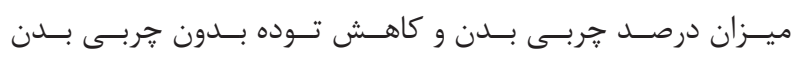

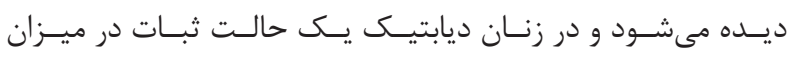

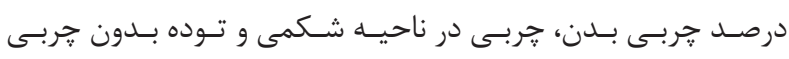

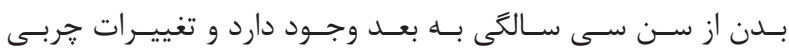

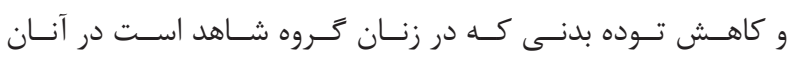

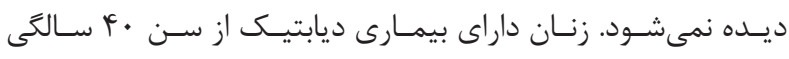

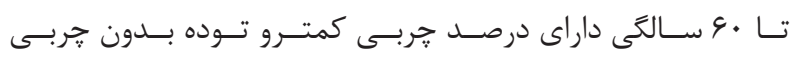

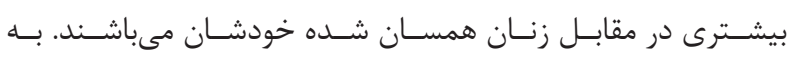

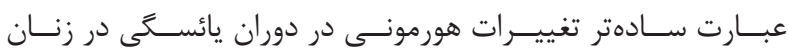

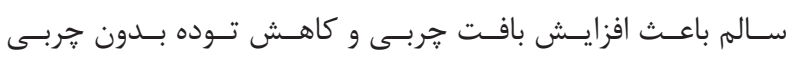

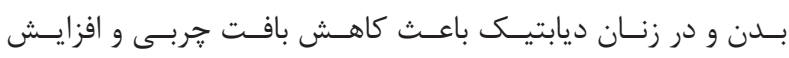

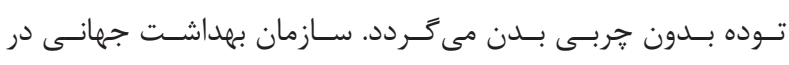

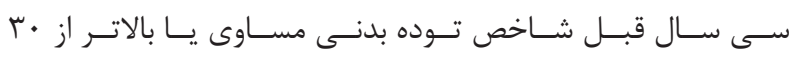

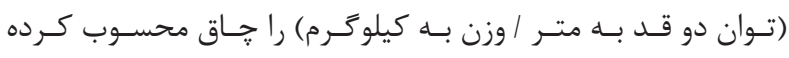

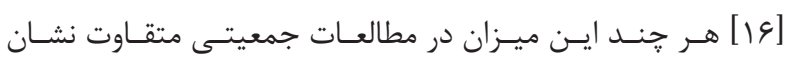

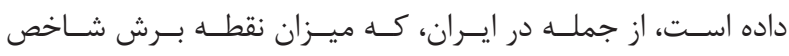

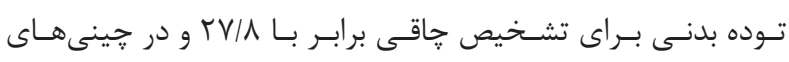

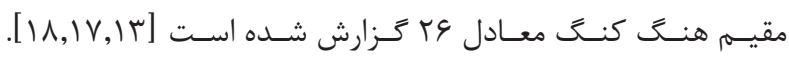

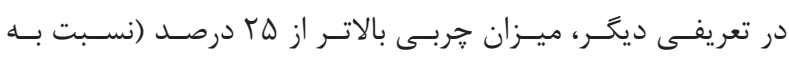

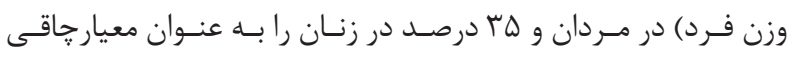

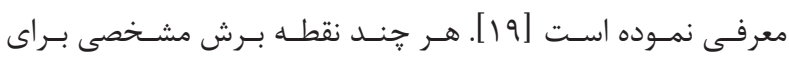

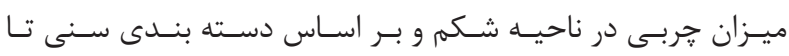

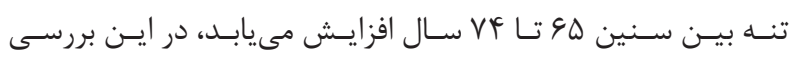

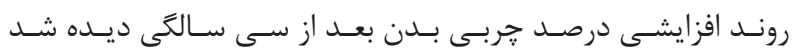

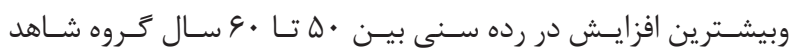

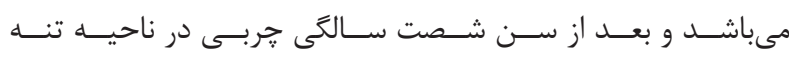

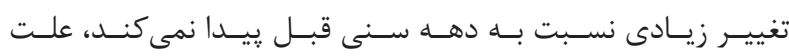

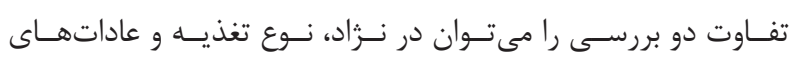

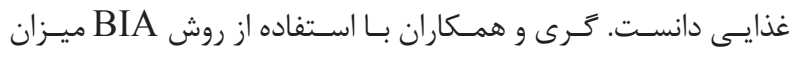

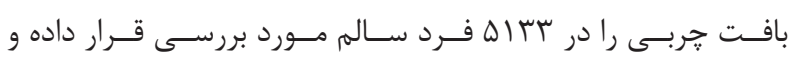

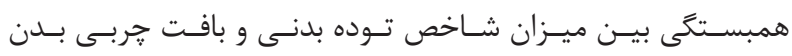

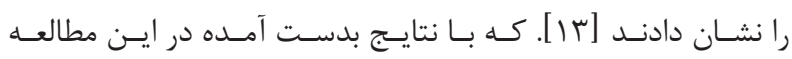

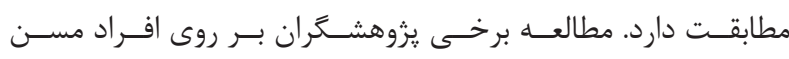

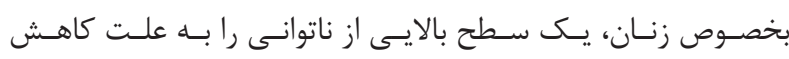

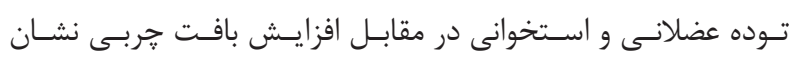

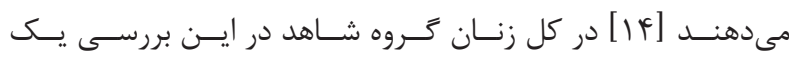

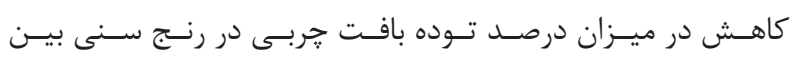

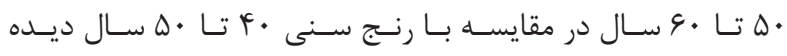

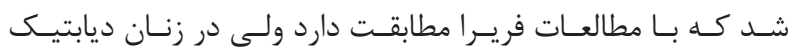

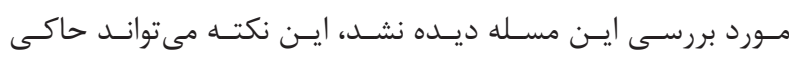

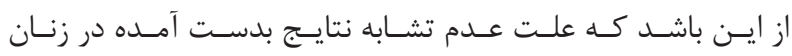

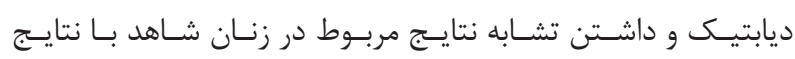

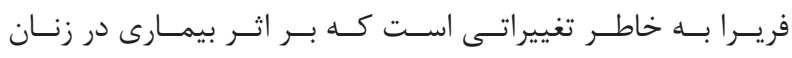

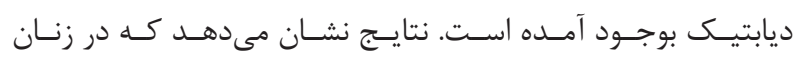

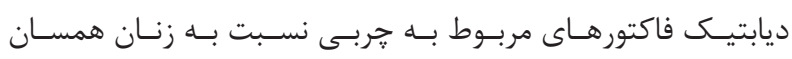

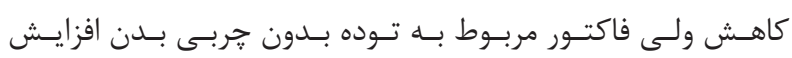

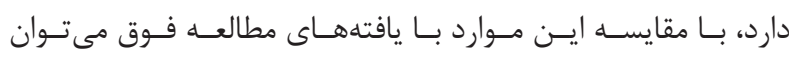

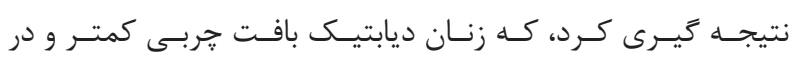

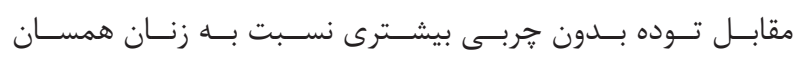

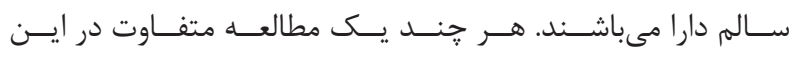

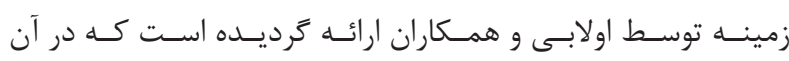

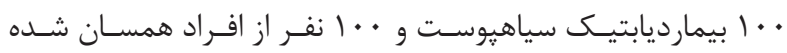

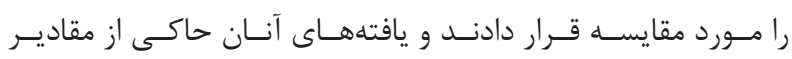

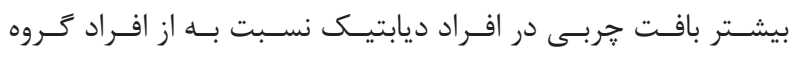

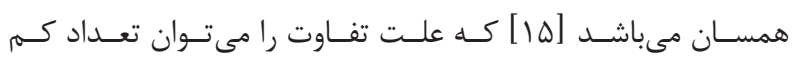

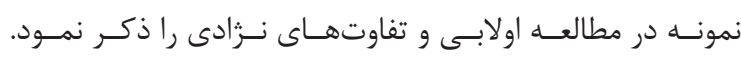

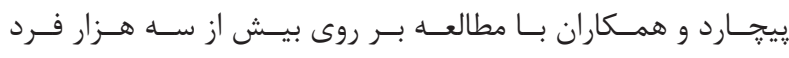

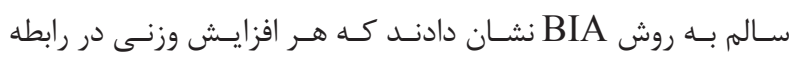

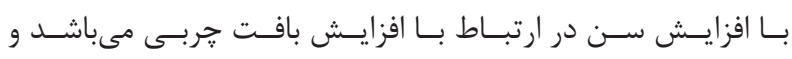

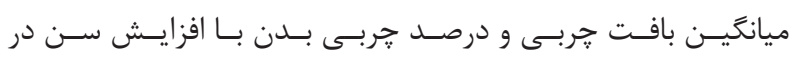

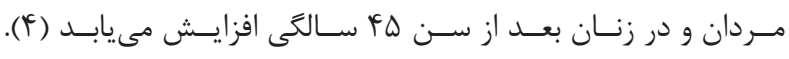

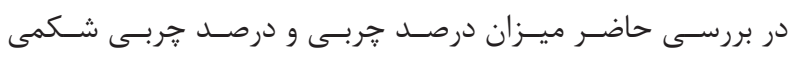

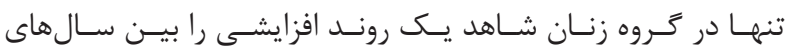




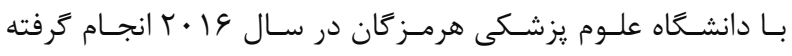

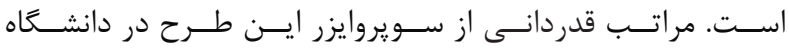

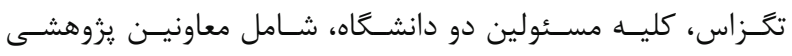

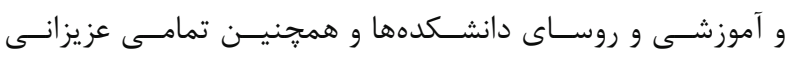

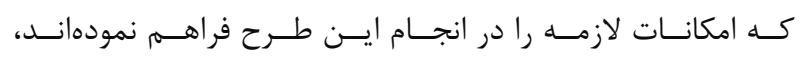

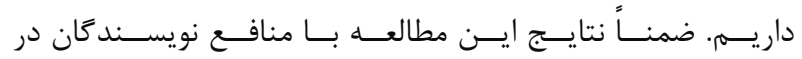

$$
\begin{aligned}
& \text { تعــارض نمىباشـــد. }
\end{aligned}
$$

\section{REFERENCES}

1 Farooq R, Amin S, Hayat Bhat M, Malik R, Ahmad Wani H, Majid S. Type 2 diabetes and metabolic syndrome - adipokine levels and effect of drugs. Gynecol Endocrinol. 2016; 5:1-4 DOI:10.1080/09513590. 2016.1207165. PMID:27705028.

2 Oguntibeju O. Diabetes mellitus - Insights and perspectives, chapter 16, a new behavioral model (Health Belief Model Combined with Two Fear Models): design, evaluation and path analysis of the role of variables in maintaining behavior, in tech publication, 2013 under CC BY 3.0 license.DOI: 10.5772/3038. site avalaible: http://www. intechopen.com/books/diabetes-mellitus-insights-and-perspectives

3 Mohtasham Amiri Z, Maddah M. [Prevalence of overweight and obesity among female medical students in guilan 2003]. Iranian J Endocrinol Metab. 2006;8(2):163-168

4 Pichard C, Kyle UG, Bracco D, Slosman DO, Morabia A, Schutz Y. Reference values of fat-free and fat masses by bioelectrical impedance analysis in 3393 healthy subjects. Nutrition. 2000;16(4):24554. PMID: 10758358 http://dx.doi.org/10.1016/S0899-9007(00)00256-2.

5 Velazquez-Alva Mdel C, Irigoyen-Camacho ME, Huerta-Huerta R, Delgadillo-Velazquez J. A comparison of dual energy X-ray absorptiometry and two bioelectrical impedance analyzers to measure body fat percentage and fat-free mass index in a group of Mexican young women . Nutr Hosp. 2014;29(5):1038-1046. DOI: 10.3305/ nh.2014.29.5.7254. PMID:24951983.

6 Wilson JP, Strauss B, Fan B, Duewer FW, Shepherd JA. Improved 4-compartment body-composition model for a clinically accessible measure of total body protein. Am J Clin Nutr. 2013;97 (3):497-504. DOI: 10.3945/ajcn.112.048074. PMID:23364008.

7 Siobhan L, Cian O, Rhoda S, Jakeman P. A comparison of dual energy $\mathrm{X}$-ray absorptiometry and bioelectrical impedance analysis to measure total and segmental body composition in healthy young adults. Eur J Appl Physiol. 2012;112(2):589-595. DOI:10.1007/s00421011-2010-4. PMID:21614505.

8 Rush EC, Chandu V, Plank LD. Prediction of fat free mass by bioimpedance analysis in migrant Asia Indian men and women: a crossvalidation study. Int J Obes (Lond). 2006;30(7):1125-31. DOI:10.1038/sj.ijo.0803230 PMID:16432545

9 Hannan WJ, Cowen SJ, Plester CE, Fearon KC, deBeau A. Comparison of bio-impedance spectroscopy and multi-frequency bio-impedance analysis for the assessment of extracellular and total body water in surgical patients. Clin Sci (Lond). 1995 Dec;89(6):651-8. PMID:8549085.

10 Earthman CP, Matthie JR, Reid PM, Harper IT, Ravussin E, Howell

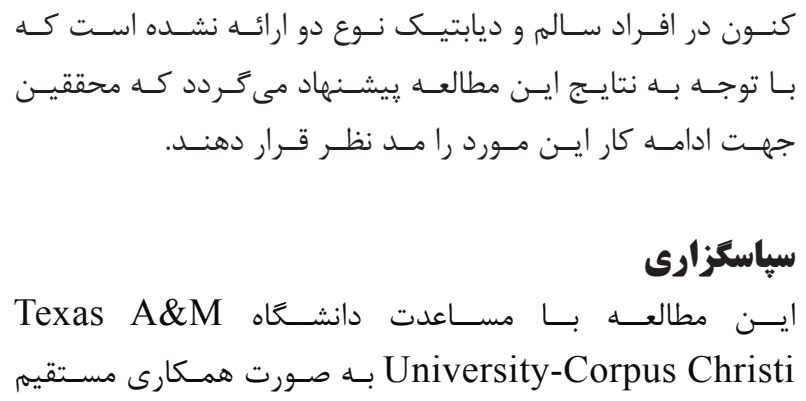

WH. A comparison of bioimpedance methods for detection of body cell mass change in HIV infection. J Appl Physiol. 2000;88(3):944956. PMID:10710390.

11 Beshyah SA, Freemantle C, Thomas E, Murphy M. Comparison of measurement of body composition by total body composition, bioimpedance analysis, and dual - energy X-ray absorptiometry in hypo pituitary adults before and during growth hormone treatment. Am J Clin Nutr 1995;61:1186-94. PMID:7762516.

12 Pedrera-Zamorano JD, Roncero-Martin R, Lavado-Garcia JM, Calderon-Garcia JF, Rey-Sanchez P, Vera V, et al. Segmental fat-free and fat mass measurements by bioelecterical impedance analysis in 2,224 healthySpanish women aged 18-85 years. Am J Hum Biol. 2015;27(4):468-74. DOI:10.1002/ajhb.22669. PMID:25452257.

13 Ko GT, Tang J, Chan JC, Sung R, Wu MM, Wai HP, et al. Lower BMI cut-off value to define obesity in Hong Kong Chinese: an analysis based on body fat assessment by bioelectrical impedance. Br J Nutr 2001;85(2):239-42. PMID: 11242492

14 Melo G, Américo, Rosa D, Fatima M. Fat-free mass in overweight and obese older women: analysis of concurrent validity of bioelectrical impedance equations. / Massa livre de gordura em idosas com sobrepeso e obesidade: análise da validade concorrente de equações de impedância bioelétrica. Braz J Kineanthropometry Human Perform. 2015;1(17)5:583.

15 Owolabi L Femi O, Adebiai S S, Danborno B, Buraimoh A. Comparative evaluation of body composition analysis in type-2 diabetes mellitus patients and healthy Nigerians using bioelectric impedance analysis technique. Nigerian J Basic Clin Sci.2016;13(1):13-18. DOI: $10.4103 / 0331-8540.176044$.

16 De Schutter A, Lavie CJ, Milani RV. The impact of obesity on risk factors and prevalence and prognosis of coronary heart diseasethe obesity paradox In obesity and obesity paradox in cardiovasculardiseases. Prog Cardiovasc Dis. 2014; Jan-Feb;56(4):401-8. DOI: 10.1016/j.pcad.2013.08.003. PMID:24438731.

17 Jahanlou A S, Kouzekanani K. The accuracy of Body Mass Index and Gallagher's classification in detecting obesity among Iranians. Iranian J Med Sci. 2016; 41(4). PMID:27365550.

18 Jahanlou A S, Kouzekanani K. Appropriate cutoff points for body mass index and waist to hip ratio for a large sample of adult population, Bandarabbas city, Iran. Zahedan J Res Medi Sci. 2016;18(8). DOI: $10.17795 /$ zjrms-7934.

19 Jahanlou A S, Kouzekanani K. [Determining the accuracy of body mass index: body composition using bioelectricalimpedance analysis(BIA)]. Arak Med Univy J (AMUJ). 2015;18(97): 26-34 


\title{
Comparison of Total Fat, Percentage of Fat, Trunk Fat, Soft Lean and Mass Waist-Hip Ratio between Diabetic Type II and Matched Non-Diabetic Iranians using Bioelectrical Impedance Analysis
}

\author{
Mojtaba Khademi ${ }^{1}$, Alireza Shahab Jahanlou ${ }^{2,}$ \\ ${ }^{1}$ Assistant Professor, Department of Internal Medicine, Faculty of Medicine, Hor- \\ mozgan University of Medical Sciences, Bandar Abbas, Iran \\ ${ }^{2}$ Associate Professor, Department of Social Medicine, Faculty of Medicine, Hormoz- \\ gan University of Medical Sciences, Bandar Abbas, Iran \\ * Corresponding author: Alireza Shahab Jahanlou, Associate Professor, Department \\ of Social Medicine, Faculty of Medicine, Hormozgan University of Medical Sciences, \\ Bandar Abbas, Iran.E-mail: jahanlu@gmail.com
}

DOI: 10.21859 /hums-24017

Received: 09.12.2016

Accepted: 15.04.2017

\section{Keywords:}

Diabetes Type 2

Body Composition

Body Fat

Fat Percentile

Soft Lean Mass

Trunk Fat

Waist-Hip Ratio

How to Cite this Article:

Khademi M, Shahab Jahanlou

A. Comparison of Total Fat, Percentage of Fat, Trunk Fat, Soft Lean and Mass Waist-Hip Ratio between Diabetic type II and Matched Non-Diabetic Iranians using Bioelectrical Impedance Analysis. Sci J Hamadan Univ Med Sci. 2017;24(1):49-55. DOI: $10.21859 /$ hums-24017

(C) 2017 Hamadan University of Medical Sciences.

\begin{abstract}
Introduction: Research shows a relationship between diabetes (type II) and obesity. Bioelectrical Impedance Analysis (BIA) is a relatively new and approved method for the measurement of body composition. The aim of this study was to compare diabetic and non-diabetic adults on the basis of waist-hip ratio, total fat, percentage of fat, trunk fat, and soft lean mass.

Methods: In this cross-sectional descriptive study, from a population of 23,395 adults, 928 diabetics (type II) were selected and matched with 928 healthy adults on the basis of age, gender, height, and weight. The analysis of data was performed by paired test and correlated samples t-test.

Results: The comparison of males in all age groups, females less than 40, and females more than 60 years old showed no statistically significant difference on the basis of all outcome measures. In females between 40 and 60 years of age, fat percentile and trunk fat were less and soft lean mass was more observed among diabetics compared to non-diabetics, and the differences were statistically significant. The waist-hip ratio was less among diabetic females in the 50-60 age group compared with the matched non-diabetics.

Conclusion: Diabetes is not related to male body composition, but is related to the body composition of females between 40 and 60 years of age, which can be due to the effects of diabetes on hormone glands during the menopause age. Among diabetic females between 50 and 60 years of age, a decrease in the waist-hip ratio can be due to the decrease in total fat and trunk fat.
\end{abstract}

